\title{
Perfil de saúde bucal de pacientes internados em uma UTI pública em Manaus/AM
}

\author{
Oral health profile of patients interned in a public ICU in Manaus/AM \\ Perfil de salud bucal de pacientes ingresados en UCI pública de Manaus/AM
}

Hannah Luiza Batista Almeida

ORCID: https://orcid.org/0000-0003-3487-8201 Universidade Paulista, Brasil

E-mail: hannah_batista@hotmail.com

Augusto Arcemiro Bittencourt

ORCID: https://orcid.org/0000-0002-2542-7655 Universidade do Estado do Amazonas, Brasil

E-mail: augustobttncrt@gmail.com

Gabriela de Sousa Soares

ORCID: https://orcid.org/0000-0002-2609-5592 Universidade Paulista, Brasil

E-mail: gabrielasoares12@hotmail.com

Nely Cristina Medeiros Caires

ORCID: https://orcid.org/0000-0003-3094-4064

Universidade Paulista, Brasil

E-mail: nelycaires@gmail.com

\begin{abstract}
Resumo
A inserção do cirurgião-dentista em equipe multidisciplinar é de suma importância e sua atuação inclui avaliação inicial do quadro bucal em que se encontra o paciente no momento de sua admissão na UTI, até a realização de procedimentos curativos e preventivos necessários à manutenção da saúde bucal. Este trabalho identificou as alterações bucais presentes em pacientes internados em Unidade de Terapia Intensiva de um Hospital Público em MANAUS/AM. Trata-se de um estudo transversal, quantitativo, de análise detalhada das informações contidas no prontuário dos pacientes internados no período de março de 2014 a março de 2015 . Dentre os 800 prontuários analisados, as alterações intrabucais mais frequentes foram: saburra lingual (94\%), cálculo dental (73\%), Queilite Angular (88\%) e Candidíase pseudomembranosa (57\%). Foram encontradas associações positivas entre Diabetes Mellitus e Queilite Angular. As Cardiopatias avaliadas no estudo estavam associadas com a presença de Cálculo dental e Candidíase pseudomembranosa. Conclui-se que a presença do cirurgião-dentista no ambiente hospitalar é de suma importância, seja no tratamento de condições pré-existentes ou na prevenção de alterações na cavidade bucal decorrentes do período da hospitalização.
\end{abstract}

Palavras-chave: Unidade de terapia intensiva; Cirurgião-dentista; Saúde bucal; Levantamento epidemiológico.

\begin{abstract}
The insertion of the dental surgeon in a multidisciplinary team is of paramount importance and its performance includes an initial assessment of the patient's oral condition upon admission to the ICU, to the performance of curative and preventive procedures necessary to maintain oral health. This study identified the oral alterations present in patients admitted to the Intensive Care Unit of a Public Hospital in MANAUS/AM. This is a cross-sectional, quantitative study of detailed analysis of the information contained in the medical records of hospitalized patients from March 2014 to March 2015. Among the 800 medical records analyzed, the most frequent intraoral changes were: tongue coating (94\%), dental calculus (73\%), Angular Cheilitis $(88 \%)$ and pseudomembranous candidiasis (57\%). Positive associations were found between Diabetes Mellitus and Angular Cheilitis. The heart diseases evaluated in the study were associated with the presence of dental calculus and pseudomembranous candidiasis. It is concluded that the presence of the dentist in the hospital environment is of paramount importance, whether in the treatment of preexisting conditions or in the prevention of changes in the oral cavity resulting from the period of hospitalization.
\end{abstract}

Keywords: Intensive care units; Dentists; Oral health; Health surveys.

\section{Resumen}

La inserción del cirujano dentista en un equipo multidisciplinario es de suma importancia y su desempeño incluye una evaluación inicial del estado bucal del paciente al ingreso a la UCI, hasta la realización de los procedimientos curativos y preventivos necesarios para mantener la salud bucal. Este estudio identificó las alteraciones bucales presentes en pacientes ingresados en la Unidad de Cuidados Intensivos de un Hospital Público de MANAUS / AM. Se trata de un estudio transversal, cuantitativo, de análisis detallado de la información contenida en las historias clínicas de los pacientes hospitalizados de marzo de 2014 a marzo de 2015. Entre las 800 historias clínicas analizadas, los 
cambios intraorales más frecuentes fueron: recubrimiento de la lengua (94\%), cálculo dental (73\%), queilitis angular $(88 \%)$ y candidiasis pseudomembranosa $(57 \%)$. Se encontraron asociaciones positivas entre diabetes mellitus y queilitis angular. Las cardiopatías evaluadas en el estudio se asociaron con la presencia de cálculos dentales y candidiasis pseudomembranosa. Se concluye que la presencia del odontólogo en el entorno hospitalario es de suma importancia, ya sea en el tratamiento de condiciones preexistentes o en la prevención de alteraciones en la cavidad bucal derivadas del período de internación.

Palabras clave: Unidad de cuidados intensivos; Cirujano dentista; Salud bucal; Encuesta epidemiológica.

\section{Introdução}

Pacientes internados em Unidade de Terapia Intensiva (UTI) muitas vezes se encontram totalmente dependentes de cuidados, portanto, impossibilitados de manter uma higienização bucal adequada, necessitando do suporte de profissionais da saúde para esta e outros tipos de tarefas. A realização da higiene bucal, visa manter a saúde bucal dos pacientes, além de prevenir injúrias e complicações sistêmicas (Rabelo et al., 2010; Blum et al., 2018; Silva et al., 2020).

A dificuldade de autocuidado do paciente em UTI é um fator predisponente a instalação de patologias bucais. É comum a redução de fluxo salivar e como consequência, ocorre acúmulo de Biofilme, cálculo dental e doença periodontal. Lesões cariosas, necrose pulpar e traumas provocados por próteses podem influenciar na condição sistêmica do paciente e agravar seu quadro clínico. (Meira et al., 2010)

A bacteremia de etiologia oral também é comum em pacientes de UTI, sendo considerada uma das causas de morbimortalidade. A higiene oral realizada com antisséptico indicado para estes pacientes é essencial para reduzir a incidência de bacteremias e pneumonia por aspiração mecânica (Sarin, 2008). Com a aspiração de secreções na cavidade oral, reduz-se o risco de aspiração de microrganismos via orofaringe (Sho et al., 2011; Alhazzani et al.,2013)

A presença do cirurgião-dentista em ambiente hospitalar tem como objetivos: o diagnóstico das alterações bucais e ser coadjuvante na terapêutica médica; realização de procedimentos emergenciais em pacientes que foram vítimas de traumatismo na face; em procedimentos preventivos e procedimentos curativos/restauradores para adequação do meio bucal, e visando proporcionar maior conforto ao paciente (Abdia, 2007).

O presente estudo irá colaborar no sentido apresentar os principais achados intra e extraoral observados em pacientes hospitalizados em uma UTI pública em MANAUS/AM, a partir de dados dos prontuários clínicos e odontológico; além de correlacionar as principais alterações orais relacionadas a doenças sistêmicas.

\section{Metodologia}

Trata-se de um estudo transversal, quantitativo (Estrela, 2018). Foram analisados 1200 prontuários de pacientes de ambos os gêneros, atendidos na UTI de um hospital público em Manaus/AM no período de março de 2014 a março de 2015. Deste total, apenas 800 prontuários atenderam aos critérios de inclusão. Os dados foram coletados de prontuários clínicos e odontológicos dos pacientes internados

Como critérios de inclusão destacamos: prontuário completo e devidamente preenchido, dados sociodemográficos, aspectos clínicos das condições de saúde bucal e sistêmica. Além destes dados foram avaliados também: dados da internação hospitalar, presença de comorbidades ou hábitos nocivos, variações da normalidade da mucosa oral e dentição, uso e necessidade de prótese dentária, qualidade da higiene bucal e necessidade de tratamento odontológico (Cota et al., 2016).

Este trabalho foi submetido e aprovado pelo CEP, sob o registro $n^{\circ}$ CAAE: 48615615.1.0000.5512.

$\mathrm{O}$ instrumento de pesquisa utilizado para a coleta de dados foi um formulário padrão para o preenchimento dos dados de interesse para o estudo. Para a análise estatística foi utilizada a associação qui-quadrado, a fim de verificar possíveis associações entre variáveis categóricas. Os programas Microsoft Excel (Microsoft Office XP) e BIOESTAT 3.0 foram utilizados para tabulação e análise dos dados. 


\section{Resultados}

Entre os dados analisado nos prontuários do paciente está o tempo de internação representado em dias no Gráfico 1.

Gráfico 1 - Tempo de internação X número de pacientes internados.

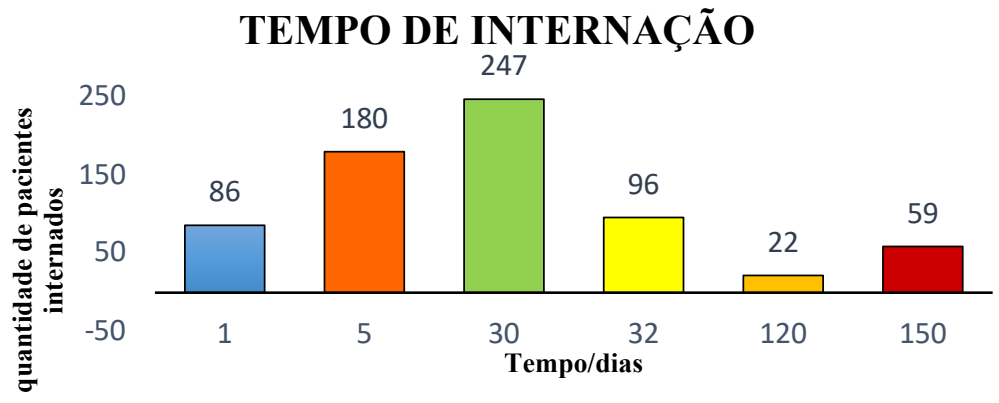

Fonte: Autores (2021).

Verifica-se que a maioria dos pacientes $(n=247)$ encontrava-se internado por um período de 30 dias na UTI em questão.

Para a detecção das alterações bucais mais frequentes ao exame intra e extraoral utilizou-se os critérios: presença de biofilme, saburra lingual, cálculo dental e queilite angular, descritos na Tabela 1.

Tabela 1. Frequência dos achados observados pela avaliação odontológica.

\begin{tabular}{lcc}
\hline \multicolumn{1}{c}{ CONDIÇÃO BUCAL } & n (800) & $\mathbf{1 0 0 \%}$ \\
\hline AVALIAÇÃO EXTRAORAL & & \\
Queilite Angular & & \\
SIM & 704 & 88 \\
NÃO & 96 & 12 \\
\hline AVALIAÇÃO INTRAORAL & & \\
Saburra Lingual & 752 & 94 \\
SIM & 48 & 6 \\
NÃO & & \\
Cálculo Dental & 584 & 73 \\
SIM & 216 & 27 \\
NÃO & & \\
Presença de Biofilme Visível & 448 & 56 \\
SIM & 352 & 44 \\
NÃO & & \\
Candidíase Pseudomembranosa & 457 & 57 \\
SIM & 343 & 43 \\
NÃO & & \\
Usuário de Prótese Total & 664 & 83 \\
SIM & 133 & 17 \\
NÃO & & \\
\hline
\end{tabular}

Fonte: Autores (2021).

Sobre a porcentagem de pacientes internados que fazem uso de Prótese Total, observa-se na Tabela 1, que a grande maioria $83 \%(n=664)$ é usuário de prótese total.

A detecção de biofilme dental visível, foi observada em mais de 50\% ( $\mathrm{n}=448)$ dos pacientes do total de 800 prontuários analisados e a presença de saburra lingual foi constatada na quase totalidade dos pacientes, sendo o total de 752 casos (Tabela 1). 
O cálculo dental, uma das condições clínicas avaliadas, pôde ser detectado em 584 pacientes (Tabela 1). A doença periodontal pode atuar como fator de disseminação de microrganismos patogênicos e predispor ao risco de desenvolver infecção nosocomial (Sannapieco, 2002).

Dos 800 prontuários analisados, 704 pacientes apresentaram Queilite Angular, durante o período de internação conforme descrito na Tabela 1.

Sobre a associação entre as condições sistêmicas e achados clínicos intraorais, observamos relação positiva entre a presença de Cálculo dental e Cardiopatia (Tabela 2).

Tabela 2 - Associação entre Cardiopatia e Cálculo Dental.

\begin{tabular}{c|cc|c|c}
\hline VARIÁVEL & \multicolumn{4}{|c}{ Cardiopatia } \\
\hline Cálculo dental & Presente & Ausente & Total & p-valor* \\
\hline SIM & 483 & 94 & 577 & \\
NÃO & 53 & 66 & 119 & 0,0001 \\
\hline
\end{tabular}

$*\left(\chi^{2}=85,502, \alpha=5 \%\right)$. Fonte: Autores (2021).

Dentre os pacientes analisados observou-se associação entre Cardiopatia e Candidíase pseudomembranosa (Tabela 3), em 457 do total de pacientes.

Tabela 3 - Associação entre Cardiopatia e Candidíase Pseudomembranosa.

\begin{tabular}{ccccc}
\hline VARIÁVEL & \multicolumn{4}{c}{ Cardiopatia } \\
\hline Candidíase pseudomembranosa & Presente & Ausente & Total & p-valor \\
\hline SIM & 457 & 80 & 537 & \multirow{2}{*}{0,0477} \\
NÃO & 215 & 23 & 238 & \\
\hline & $*\left(\chi^{2}=85,502, \alpha=5 \%\right)$ & Fonte: Autores $(2021)$. &
\end{tabular}

Em relação à associação entre Quelite Angular e Diabetes Mellitus tipo 2, constata-se na Tabela 4, uma associação presente em 333 pacientes.

Tabela 4 - Associação entre Diabetes Mellitus tipo 2 e Quelite Angular.

\begin{tabular}{ccccc}
\hline VARIÁVEL & \multicolumn{4}{c}{ Diabetes Mellitus tipo 2 } \\
\hline Quelite angular & Presente & Ausente & Total & p-valor \\
\hline SIM & 333 & 38 & 371 & 0,0001 \\
NÃO & 30 & 17 & 47 & \\
\hline \multirow{2}{*}{$*\left(\chi^{2}=85,502, \alpha=5 \%\right)$} & Fonte: Autores $(2021)$.
\end{tabular}

\section{Discussão}

A avaliação das condições orais em pacientes internados em UTI é de extrema importância. Sabe-se que as comorbidades e complicações que levam esses pacientes à internação se somam às possibilidades de adquirir infecção nosocomial durante períodos prolongados de internação. Além disto, o paciente pode sofrer alterações orais decorrentes de doenças sistêmicas ou do uso de medicamentos e ventilação mecânica invasiva (Schlesener et al., 2012; Kiyoshi-Teo \& Blegen, 2015). 
Tendo em vista que, na maioria dos hospitais brasileiros, a presença de cirurgião-dentista em equipes multidisciplinares que atuam em UTIs ainda é relativamente pequena, faz-se necessário a publicação de dados sobre o perfil de saúde bucal destes pacientes, a fim de tornar visível e notório os benefícios da atuação odontológica em ambiente hospitalar e UTIs.

Este trabalho avaliou 800 prontuários incluídos no estudo dos 1200 pacientes internados e assistidos pela equipe odontológica no período de março de 2014 a março de 2015 em um hospital público em Manaus/AM. Notamos que no estudo em questão o período de internação dos pacientes foi longo (mais de 30 dias) embora os cuidados de higiene oral devam ser instituídos logo após a internação do paciente em UTI. A avaliação cuidadosa de condições odontológicas pré-existentes e a instituição de protocolo de higiene oral são fundamentais na prevenção de infecção nosocomial. Estudos mostraram que a quantidade de biofilme em pacientes internados em UTI, aumenta com o tempo de internação, paralelamente também ocorre aumento da diversidade de patógenos respiratórios que colonizam o biofilme bucal (Gomes \& Esteves, 2012).

Dentre as condições observadas chama-nos à atenção, o número de pacientes edêntulos e usuários de prótese total que correspondeu a mais de $80 \%(n=664)$ do total de pacientes avaliados. Essa constatação permite revelar que a presença de desdentados totais ainda é predominante no Brasil como um todo. E paralelamente aos avanços técnico-científicos em odontologia, ainda coexistem a exclusão social e dificuldades de acessos aos serviços odontológicos, e resolutividade nas ações de promoção de saúde, prevenção e manejo das doenças e agravos em saúde bucal. Associado a isto, o fato de os pacientes usarem a prótese total em ambiente hospitalar sem os devidos cuidados de higiene e manutenção, é comumente relatado ao acúmulo de biofilme e cálculo dental, podendo evoluir para Candidíase e Queilite Angular (Gebran \& Gebert, 2002; Stramandinoli et al., 2010).

O biofilme dental é fator etiológico primário para o surgimento da doença periodontal. Mais ainda, em relação a pacientes internados em UTI, os dentes e gengivas representam um reservatório para os patógenos respiratórios e as bactérias fixadas e acumuladas no biofilme dental podem se deslocar para a orofaringe e colonizar o trato respiratório. Como consequência os pacientes internados neste ambiente apresentar um risco maior de desenvolver a Pneumonia Nosocomial. (Cota et al., 2016). Em nosso estudo 56\% dos pacientes analisados possuíam biofilme dental visível, o que pode indicar falhas no processo de higiene oral e que precisam ser corrigidos através da orientação da equipe de Enfermagem, pelo cirurgiãodentista, sobre a realização/manutenção da higiene oral.

A presença de saburra lingual foi detectada na quase totalidade dos pacientes ( $93 \%$ ), o que indica falhas na higiene oral e higiene da língua do paciente. A presença de saburra lingual, em conjunto com o biofilme dental, acarreta acúmulo de microrganismos patogênicos e possibilidade de infecção nosocomial. A melhor forma de promover a saúde bucal é controlar o biofilme dental e a saburra lingual, por meio do uso de meios mecânicos e químicos. Os meios mecânicos consistem na escovação, utilizando fio dental, escovas inter-proximais e raspadores de língua. Para o paciente com incapacidade para executar o controle mecânico do biofilme, está indicado o meio químico de controle (Sannapieco, 2002), associado a remoção mecânica da saburra lingual.

Sobre o cálculo dental, foi detectado em 584 pacientes, principalmente nos dentes anteriores inferiores e até na prótese total de um dos pacientes. Estes dados corroboram para o fato de que a higiene oral destes pacientes era bem aquém do esperado e necessário. Alguns autores relataram que a doença periodontal pode atuar como fator de disseminação de microrganismos patogênicos e predispor ao risco de desenvolver infecção nosocomial (Sannapieco, 2002; Stramandinoli et al., 2010).

A candidíase oral é uma condição frequente entre pacientes internados em unidades de Terapia intensiva, devido a alterações locais e sistêmicas que modificam a microbiota oral. Entre as alterações locais estão o uso de aparelhos protéticos e ortodônticos, tabagismo, hipossalivação, mudança de hábitos alimentares e higiene oral deficiente. Há quatro manifestações 
básicas da Candidíase: pseudomembranosa, eritematosa, crônica hiperplásica e queilite angular (Gebran \& Gebert, 2002; Preshaw et al., 2012; Batista et al., 2014; Santos-Paul et al., 2015). A queilite angular embora detectada extra oralmente, estava associada à presença de Candidíase oral, o que auxiliou na confirmação do diagnóstico em $88 \%$ dos pacientes analisados.

A correlação entre os achados acima citados e doenças sistêmicas como Diabetes Mellitus tipo 2 e Cardiopatias, também foram objeto deste estudo. Vale ressaltar que indivíduos portadores de doenças sistêmicas, como o diabetes mellitus e Cardiopatias, são propensos a desenvolver complicações sistêmicas crônicas. O Diabetes Mellitus tipo 2 está relacionado à evolução de tais alterações bucais e podem interferir no plano de tratamento odontológico, aumentando o tempo de internação e os custos hospitalares. Tanto nas cardiopatias quanto no Diabetes Mellitus, é essencialmente recomendável que o profissional de Odontologia inserido em equipes multidisciplinares que atuam na UTI, realize avaliações odontológicas juntamente com a equipe médica, de modo a dimensionar as condições sistêmicas e os agravos bucais e optar pelo melhor tipo de atendimento demandado.

Já bem evidenciado na literatura, as doenças periodontais são mais prevalentes e apresentam maior severidade em portadores de Diabetes mellitus. Observa-se clinicamente, maior perda de inserção, maior reabsorção óssea e maior sangramento gengival à sondagem que pacientes não diabéticos. Além da periodontite, a Candidíase representa uma das manifestações orais mais observadas em diabéticos descompensados (Yamashita et al., 2013; Stramandinoli et al., 2010; Galhardo et al., 2020).

Há um fator de risco aumentado para a cárie dental em consequência da hipossalivação e alta concentração de glicose na saliva. Neste caso, a equipe odontológica deve implementar protocolos preventivos e curativos quando necessários. Quanto ao ressecamento labial, se faz necessário a hidratação com ácidos graxos essenciais (AGE), Despantenol ou óleo de coco, a fim de prevenir a formação de lesões e contaminação destas por fungos $(20,21,22)$.

\section{Considerações Finais}

A importância do cirurgião-dentista na equipe multidisciplinar atuando na UTI é fundamental para a melhoria da qualidade de vida dos pacientes internados; uma vez que estes se encontram totalmente dependentes de cuidados e impossibilidatos de manter a higiene bucal adequada. O monitoramento e a descontaminação da cavidade oral dos pacientes em UTI por profissionais qualificados são a chave para a redução da colonização pulmonar por patógenos orais e, consequentemente, da redução da incidência de infecções nosocomiais. Esperamos em trabalhos futuros, evidenciar o perfil de saúde bucal de pacientes internados em UTI, quando existe uma rotina de atendimento do cirurgião-dentista devidamente inserido na equipe multidisciplinar.

\section{Referências}

Abidia, R. F. Oral Care in the Intensive Care Unit: A Review. (2007). The Journal of Contemporary Dental Practice, 8(1):76-82.

Alhazzani, W., Smith, O., Muscedere, J., Medd, J., \& Cook, D. Toothbrushing for Critically Ill Mechanically Ventilated Patients: A Systematic Review and Meta-Analysis of Randomized Trials Evaluating Ventilator-Associated Pneumonia (2013). Critical care medicine, 41 (2): $646-55$.

Batista, S. A., Siqueira, J. S. S., Silva Jr, A., Ferreira, M. F., \& Agostini, M. (2014). Alterações orais em pacientes internados em unidades de terapia intensiva. Revista Brasileira de Odontologia, 71(2):156-9.

Blum, D. F., Silva, J. A., Baeder, F. M., \& Della Bona, A. A. (2018). Atuação da Odontologia em unidades de terapia intensiva no Brasil. Revista Brasileira de Terapia Intensiva, 30(3):327-332.

Costa, D. C., Saldanha, K. F., Sousa, A. S., \& Gaetti-Jardim, E. C. (2016). Perfil de saúde bucal dos pacientes internados no Hospital Universitário Maria Aparecida Pedrossian, Campo Grande (MS). Archives of Health Investigation, 5(2): 70-77.

Estrela, C. (2018). Metodologia cientifica: ciência, ensino, pesquisa. (3 ${ }^{\mathrm{a}} \mathrm{ed}$.): Artes Médicas.

Galhardo, L., Ruivo, G. F., Ferreira, T. F., Santos, F. O., Sendyk, W. R., Kim, Y. J., Martins, F. M., Boaventura, R. M., Braz-Silva, P. H., \& Pallos, D. Lesões bucais em pacientes hospitalizados em unidade de terapia intensiva. (2020). Research, Society and Development, $9(8)$ : e629985945. 
Research, Society and Development, v. 10, n. 8, e1010817020, 2021

(CC BY 4.0) | ISSN 2525-3409 | DOI: http://dx.doi.org/10.33448/rsd-v10i8.17020

Gebran, M. P., \& Gebert, A. P. O. (2002). Controle químico e mecânico de placa bacteriana. Tuiuti: Ciência e Cultura, 3(26):45-58.

Gomes, S. F., \& Esteves, M. C. L. (2012). Atuação do cirurgião-dentista na UTI: um novo paradigma. Revista Brasileira de Odontologia, 69 (1): 67-70.

Kiyoshi-Teo, H., \& Blegen, M. (2015). Influence of Institutional Guidelines on Oral Hygiene Practices in Intensive Care Units. American Journal of Critical Care, 24(4):309-18.

Mariani, T. R., Silva, S. O., \& Carli, J. P. (2016). Prevalência de candidose bucal em pacientes hospitalizados e avaliação dos fatores de risco. Salusvita, 35(3): 379-395.

Meira, S. C. R., Oliveira, C. A. S., Ramos, I. J. M. (2010). A importância da participação do cirurgião-dentista na equipe multiprofissional hospitalar. $9^{a}$ edição do prêmio SINOG de Odontologia. Curso de Odontologia do Centro Universitário Newton Paiva, Belo Horizonte/MG.

Preshaw, P. M., Alba, A. L., Herrera, D., Jepsen, S., Konstantidinis, A., Makrilakis, K., R. T. (2012). Periodontitis and diabetes: a twoway relationship. Diabetologia, 55(1):21-31.

Rabelo, G. D., Queiroz, C. I., \& Santos, P. S. S. (2010). Atendimento odontológico ao Paciente em Unidade de Terapia Intensiva. Arquivos Médicos Dos Hospitais e da Faculdade de Ciências Médicas da Santa Casa De São Paulo, 55(2): 67-70.

Sannapieco, F. A. (2002). Relação entre Doença Periodontal e Doenças Respiratórias, em: Rose L. E., Genco, R. J., Mealy, B. L., et al - Medicina Periodontal. São Paulo: Santos.

Santos-Paul, M. A., Neves, I. L., Neves, R. S., \& Ramires, J. A. (2015). Local anesthesia with epinephrine is safe and effective for oral surgery in patients with type 2 diabetes mellitus and coronary disease: a prospective randomized study. Clinics, 70(3):185-9.

Sarin, J., Balasubramaniam, R. Corcoran, A. M., Laudenbach, J. M., \& Stoopler, E. T. (2008). Reducing the Risk of Aspiration Pneumonia among Elderly Patients in Long-Term Care Facilities through oral Heath interventions. Journal of the American Medical Directors Association, 9 (2): $128-35$.

Schlesener, V. R. F., Rosa, U. D., \& Raupp, S. M. M. (2012). O cuidado com a saúde bucal de pacientes em UTI. Cinergis, $13(1): 73-7$.

Silva, E. A., Silva, A. B. S., Machado, I. F., Lins Irmão Bisneto, J. S., Santos, I. I. S., \& Cota, A.L.S. (2020). A importância da atuação do cirurgião dentista na unidade de terapia intensiva: revisão integrativa. Research, Society and Development, 9(6): e164962011.

Soh, K. L., Soh, K. G., Japar, S., Raman, R. A., \& Davidson, P. M. (2011). A cross-sectional study on nurses oral care practice for mechanically ventilated patients in Malaysia. Journal of Clinical Nursing, 20(5-6): 733-42.

StramandinolI, R. T., Souza, P. H. C., Westphalen, F. H., Bisinelli, J. C. Ignácio, S. A., \& Yurgel, L. S. (2010). Prevalência de candidose bucal em pacientes hospitalizados e avaliação dos fatores de risco. Revista Sul-Brasileira de Odontologia - RSBO, 7(1):66-72

Yamashita, J. M., Moura-Grec, P. G., Capelari, M. M., Sales- Peres, A., \& Sales-Peres, S. H. C. (2013). Manifestações bucais em pacientes portadores de Diabetes Mellitus: uma revisão sistemática. Revista de Odontologia da UNESP, 42(3):211-220. 